\title{
Article
}

\section{Do clinical guidelines guide clinical practice in stroke rehabilitation? An international survey of health professionals}

Lynch, Elizabeth A., Connell, Louise Anne, Carvalho, Lilian B. and Bird, Marie-Louise

Available at http://clok.uclan.ac.uk/37043/

Lynch, Elizabeth A., Connell, Louise Anne ORCID: 0000-0002-0629-2919, Carvalho, Lilian B. and Bird, Marie-Louise (2021) Do clinical guidelines guide clinical practice in stroke rehabilitation? An international survey of health professionals. Disability and Rehabilitation . ISSN 0963-8288

It is advisable to refer to the publisher's version if you intend to cite from the work. http://dx.doi.org/10.1080/09638288.2021.1891304

For more information about UCLan's research in this area go to http://www.uclan.ac.uk/researchgroups/ and search for <name of research Group>.

For information about Research generally at UCLan please go to http://www.uclan.ac.uk/research/

All outputs in CLoK are protected by Intellectual Property Rights law, including Copyright law. Copyright, IPR and Moral Rights for the works on this site are retained by the individual authors and/or other copyright owners. Terms and conditions for use of this material are defined in the policies page. 


\section{Do clinical guidelines guide clinical practice in stroke rehabilitation? An international survey of health professionals}

Purpose: To identify health professionals' awareness of stroke rehabilitation guidelines, and factors perceived to influence guideline use internationally.

Methods: Online survey study. Open-ended responses were thematically analysed, guided by the Consolidated Framework for Implementation Research.

Results: Data from 833 respondents from 30 countries were included. Locally developed guidelines were available in 22 countries represented in the sample. Respondents from highincome countries were more aware of local guidelines compared with respondents from lowand middle-income countries.

Local contextual factors such as management support and a culture of valuing evidence-based practice were reported to positively influence guideline use, whereas inadequate time and shortages of skilled staff inhibited the delivery of guideline-recommended care. Processes reported to improve guideline use included education, training, formation of workgroups and audit-feedback cycles. Broader contextual factors included accountability (or lack thereof) of health professionals to deliver rehabilitation consistent with guideline recommendations.

Conclusions: While many health professionals were aware of clinical guidelines, they identified multiple barriers to their implementation. Efforts should be made to raise awareness of local guidelines in low- and middle-income countries. More attention should be paid to addressing local contextual factors to improve guideline use internationally, going beyond traditional strategies focused on individual health professionals. 


\section{Do clinical guidelines guide clinical practice in stroke rehabilitation? An international survey of health professionals}

\section{Introduction}

On the international stage, stroke is a leading cause of adult disability [1]. While the evidence base for effective stroke rehabilitation interventions is growing exponentially[2], data from different world regions consistently indicate that many evidence-based stroke rehabilitation interventions are not routinely applied in clinical practice.[3-5] In short, this means that many stroke survivors do not receive interventions that have been proven to reduce disability and optimise function, which may contribute to unnecessary suffering and added burden to health systems.[6]

In an effort to enhance the use of evidence-based treatments in clinical practice, health authorities in many countries and regions have developed clinical practice guidelines (hereafter called "guidelines"), which are "systematically developed statements to assist practitioner and patient decisions about appropriate health care for specific clinical circumstances."[7] Rigorous guideline development is contingent upon having access to sufficient people and resources to identify the guideline scope, identify and review the published literature, synthesise and rate the evidence, generate then review practical recommendations and disseminate the final product.[8] Accordingly, the majority of stroke rehabilitation guidelines have been developed in high income countries (HICs).[9] Rather than develop their own guidelines from scratch, some middle-income countries such as the Philippines and South Africa, have opted to adapt stroke rehabilitation guidelines developed in HICs and contextualise these for local use.[10,11] This approach has been recommended by international experts to improve access to evidence-based stroke rehabilitation in low- and middle-income countries (LMICs).[12] 
The Global Burden of Disease Stroke Collaborators have highlighted the global need for appropriate acute and long-term (including rehabilitation) care for people with stroke.[1] While there is growing availability of locally relevant stroke rehabilitation guidelines in HICs, little is known about international adherence to recommended rehabilitation care for people with stroke. We were interested in gaining a global overview of the factors that influenced the use of stroke rehabilitation guidelines, as a first step towards informing international efforts to enhance rehabilitation delivery to people with stroke.

\section{Aims}

1. To evaluate whether health professionals working in stroke rehabilitation in different world regions were aware of, and had access to, guidelines for stroke rehabilitation.

2. To identify factors that influenced use of stroke rehabilitation guidelines in different world regions.

\section{Methods}

\section{Study design and procedure:}

This survey study originates from work conducted by the international Knowledge Translation (KT) Working Group, convened as part of the second Stroke Recovery and Rehabilitation Roundtable.[13, 14]

The KT Working Group and its international advisory group, which consisted of representatives of eight professions from 13 countries in 6 continents, identified potential participants and professional groups to assist with survey dissemination. Invitations to participate in an online survey were then sent to health professionals working in stroke rehabilitation via email and advertised on social media through these international and local networks. People were eligible to participate in the survey if they were health professionals of any discipline working in the field of stroke rehabilitation. The study received ethical 
approval (UBC HREC Approval number H1801633). Survey data were collected between 13th June and 26th September 2018.

\section{The Survey Instrument}

The KT Working Group developed an online survey through a collaborative process, which has been described previously.[14] The survey included 8 items regarding participant demographics and 3 closed- and 6 open-ended questions to gather perspectives from healthcare providers about access to clinical guidelines, factors influencing use of guidelines and evidence-based practice, and KT priorities for stroke rehabilitation. The survey was developed in the English language, was translated into Chinese and Portuguese by native speakers and was delivered through the Qualtrics platform. Data were exported from Qualtrics to Excel. Responses in Portuguese and Chinese were translated into English prior to analysis.

Responses to the questions regarding KT priorities have been reported elsewhere.[14] This paper reports data from the following survey questions:

Do you have clinical practice rehabilitation guidelines in your country?

a. If yes, please specify

b. If no, are guidelines from another country commonly used?

2. What facilitates the use of guidelines in your local region?

3. What are the barriers to guideline use?

4. What other things improve the uptake of Evidence Based Practice in your local region?

\section{Data analysis}

To be included in the analysis, respondents needed to provide data about whether their country had guidelines and nominate the country where they worked (or have location identifiable through the IP address). 
Demographic data and the responses to the presence of national guidelines were analysed descriptively.

Data from the open-ended questions regarding barriers and enablers to guideline use were analysed deductively using the Consolidated Framework for Implementation Research (CFIR). Reponses were coded into the following five domains: intervention characteristics, outer setting, inner setting, individual characteristics and process.[15] Two researchers coded data about barriers (LAC, MLB) and two others coded facilitator data (EL, LBC). The researchers (with backgrounds in physiotherapy and medicine, and clinical or academic experience in Australia, Brazil, Canada and the United Kingdom [UK]) met over videoconference to check the consistency of coding systems and to discuss the emergent findings as coding was underway, and when coding was complete.

Availability of guidelines in each country listed by participants was cross-checked by author LBC who performed an online search in English or in the country's primary language, looking for national stroke rehabilitation guidelines. The income level of each country as classified by the World Bank was recorded.[16]

\section{Results}

Due to the chosen method of survey dissemination, where professional groups and clinical networks were asked to invite health professionals to participate in the survey, the response rate could not be calculated.

833 respondents completed question 1 (presence of local guidelines) and just over half of these respondents $(n=468)$ provided data to the open-ended questions about barriers or enablers to guideline use or other factors that improve the use of evidence-based practice. Demographic data were inconsistently provided; country of residence $(n=833$, often able to be calculated from IP address if not provided by the respondent) and health discipline $(\mathrm{n}=451)$ were the items most commonly available. 


\section{Presence and awareness of local stroke rehabilitation guidelines}

833 respondents from 30 countries provided data about whether they had local stroke rehabilitation guidelines in their country. Just over half of the respondents were from HICs (453 respondents from 22 HICs; 285 respondents from 4 upper middle-income countries; 49 respondents from 3 lower middle-income countries; 1 respondent from 1 low income country), see Table 1 . The majority of respondents were from China ( $n=216,26 \%$ of respondents), Australia $(n=156,19 \%)$, the $\mathrm{UK}(\mathrm{n}=125,15 \%)$, Canada $(\mathrm{n}=85,10 \%)$, or Brazil $(\mathrm{n}=64,8 \%)$. Of the 451 respondents who provided their professional discipline, the largest proportion were physiotherapists $(n=181)$, followed by speech pathologists $(n=71)$, occupational therapists $(n=66)$ and physicians $(n=57)$. More detail is supplied in Supplementary Table 1.

Our online search identified national stroke rehabilitation guidelines for 22 of the 30 countries represented in our sample.[17-40] Fourteen survey responses from the eight countries without identified guidelines (Belgium, Greece, Nigeria, Oman, Portugal, Saudi Arabia, Sudan, United Arab Emirates) confirmed the lack of national guidelines, but one of the seven respondents from Nigeria reported awareness of national guidelines which were not located in our search. Locally endorsed guidelines were identified for 15 of the 21 HICs represented in the sample, for all 4 upper middle-income countries, for 2 of the 3 lowermiddle income countries, but none were identified for the 1 low-income country.

\section{[insert Table 1 here]}

When looking at data from countries where ten or more responses were collected, most respondents from HICs reported that their country had stroke rehabilitation guidelines; at least 98 percent of the respondents from Australia, Canada, Ireland, Sweden and the UK reported having national guidelines. In comparison, awareness of national guidelines from respondents from the United States of America (USA) was not so strong (46 respondents, 
93\% aware of USA guidelines) and awareness in Singapore was particularly low (30 respondents, $33 \%$ aware of Singaporean guidelines).

Awareness of national guidelines from respondents from LMICs were mixed, with inconsistencies reported within countries such as China (216 respondents, 85\% aware of Chinese guidelines), Brazil (64 respondents, 72\% aware of Brazilian guidelines) and India (38 respondents, 39\% aware of Indian guidelines).

When respondents reported that their country did not have stroke rehabilitation guidelines, a small proportion (40 respondents) reported using guidelines from other countries, most often USA, Canada, UK and Australia.

\section{Factors influencing use of stroke rehabilitation guidelines}

468 respondents answered questions about barriers and enablers to guideline use, or other factors that improve the uptake of evidence-based practice. The majority of respondents to these questions were from Australia ( $n=101,22 \%$ of respondents to open-ended questions), the UK $(n=72,15 \%)$, Canada $(n=65,14 \%)$, China $(n=65,14 \%)$ and Brazil $(n=46,10 \%)$.

Responses are presented below as per their mapping to the domains and constructs of the CFIR, in order of numbers of responses mapped to each of the five domains, with exemplar quotes presented for each domain. The domain with the most data mapped to it for both facilitators (305 responses) and barriers (403 responses) was "Inner setting”.

\section{Inner setting:}

The systems and supports in place in respondents' places of work appeared to strongly influence guideline use. Being part of a clinical team that valued evidence-based practice and worked together collectively was reported to enable use of guidelines and evidence-based practice.

"Language used by team ('according to the clinical guidelines... '.) prompts me”" [speech-language pathologist, Australia] 
"We have a culture of development... Our clinicians value stroke best practice, we select annual objectives in team (manager, clinician and patient)" [profession undocumented, Canada]

Support and interest from management, in terms of providing dedicated time to plan, do and evaluate implementation projects and providing access to professional development opportunities such as education and training were consistently reported to facilitate care compliant with guideline recommendations.

"Support from upper administration in terms of continuing education and implementation of best practice" [speech-language pathologist, Canada]

Conversely, respondents reported a lack of time (to read, plan or implement), staffing issues (low numbers, lack of skill, lack of experience) and inaccessibility were common barriers to use of stroke rehabilitation guidelines.

"[lack of] Time to keep up with the guidelines and to implement them, lack of access to professional development being in a regional area" [nurse, Australia]

"Scarcity of stroke rehabilitation staff" [nurse, China]

[Poor] Availability of guidelines, very few experts" [physiotherapist, Nigeria]

Increasing clinical caseloads and increasing administrative requirements were other barriers commonly reported.

"Time constraints and higher patient load" [physiotherapist, India]

"Increased administration demands ...takes time away from clinical work" [speechlanguage pathologist, Ireland]

Process:

Most data mapped to process were relevant to supporting guideline use (188 facilitator responses, 5 barrier responses). Respondents commonly cited participation in education and training activities as facilitating guideline use. 
"Education on updates and changes to guidelines, facilitated by online learning \& team discussions" [occupational therapist, Canada]

“Relevant training and learning” nurse, China]

When implementing guideline recommendations, respondents described the benefit of forming dedicated workgroups, having people assigned to lead or facilitate implementation projects and having implementation mentors.

"Designated clinical practice leads, dedicated time and experts in knowledge translation" [physiotherapist, Canada]

"Our local organisation using results from the National register trying to identify weak areas... we will now focus on... appointing one of the nurses to be a 'spokesperson' to provide information on guidelines and to feedback when someone is not following guidelines” [speech-language pathologist, Sweden]

A lack of engagement by key members of the team, such as physicians, could be a barrier. Creating momentum and having a coordinated approach were also seen as important. Reflecting and evaluating through the use of audit and feedback was seen as an enabler by a large number of respondents, although when feedback was focussed on indicators other than guideline implementation (such as length of stay), this was reported as a barrier by other respondents.

"Audit culture does not always promote guideline implementation but can focus only on meeting audit standards" [nurse, UK]

\section{Individual characteristics:}

The majority of data mapped to individual characteristics were about barriers to guideline use (115 responses vs 35 facilitator responses). Respondents commonly reported people (either themselves or their colleagues) were unaware of guidelines, lacked awareness of evidence, or lacked knowledge, skills and confidence to implement evidence. 
"Not enough awareness" [physician, USA]

"Unawareness regarding the rehabilitation guidelines" [physiotherapist, India]

"Most common problem is therapists not knowing how to [implement] an

intervention...due to lack of knowledge and skills, or confidence” [occupational

therapist, Australia]

The unwillingness of some individuals to change practice or "knowing better" than the guidelines from health professionals' own clinical practice was also reported.

"Lack of interest and care about research findings- 'I know from experience what works "'[physiotherapist, Sweden]

In contrast, an individual's motivation or dedication to deliver evidence-based practice could be a positive influence.

"My own desire to give the patient the best evidence practice" [physiotherapist, Sweden]

Several respondents identified that embedding evidence-based practice in the curricula of health professionals' education programs could facilitate its use.

\section{Intervention characteristics:}

The nature of the guidelines was important, with more barriers (126 responses) reported than facilitators (43 responses). Ease of applying recommendations and step-by-step guides facilitated guideline use; however, commonly reported barriers were guidelines that were hard to read, hard to understand or lacked specific recommendations. Needing to adapt guidelines to local context or local language was an additional barrier for some respondents.

The guidelines are often vague and don't necessarily provide specifics regarding applying the evidence [physiotherapist, Canada] 
"Guidelines are mostly in English which makes access a little difficult for a few professionals. Also, the length of guidelines can be quite overwhelming” [physician, Brazil]

Since we follow foreign guidelines, these do not necessarily attend the need \& reality of our country [speech-language pathologist, Brazil]

Trust in the evidence upon which the guidelines were based was reported to be a facilitator; whereas considering the guidelines to be out-of-date or not reflecting local developments were barriers.

Guidelines can ...lag behind locally relevant developments [physiotherapist, UK] Guidelines are not comprehensive enough for people that work with rehabilitation. They are not in-depth and they are currently 5-years old.[physician, Brazil]

Additional barriers reported included that guidelines do not always address specific clinical presentations such as visual impairments, and recommendations were not always considered feasible to implement, particularly if they were contingent on having specific equipment or staffing levels.

"The contemporary evidenced approaches are often not feasible in the real clinical practice” [occupational therapist, Taiwan]

There were divergent views about the benefit of adhering to guideline recommendations; some respondents believed patients would have better clinical outcomes if stroke guidelines were used:

"Streamline resources, improve outcomes and service to patients" [physiotherapist, Singapore]

whereas others reported that applying recommendations from guidelines would take longer than providing usual care for no added benefit. 
The application of a new intervention may take more time than interventions already

known [physiotherapist, Canada]

No benefit [physiotherapist, USA]

Outer setting:

Similar numbers of responses regarding barriers $(n=46)$ and facilitators $(n=57)$ were mapped to the outer setting domain. The most frequently reported barrier to guideline use was lack of oversight or mandate by professional bodies or health service managers to deliver care recommended in guidelines; respondents reported that health professionals were not required to be accountable for delivering evidence-based care or to account for patients' clinical progress.

"There is no legislation that request the use of guidelines, therefore it is individual professional decision, whether to use guidelines or not in clinical practice" [physician, Latvia]

"Lack of accountability checks of health professionals regarding patient progress" [physiotherapist, India]

In contrast, policy support, professional standards and professional board recommendations were perceived to support guideline use.

“Drivers from professional standards/policies and procedures, e.g. requirement for [evidence-based practice] in order to meet professional standard and registration, to be demonstrated at annual staff appraisal " [physiotherapist, UK]

When patients were aware of evidence-based care, and advocated for its use, this facilitated the use of guidelines. However, some respondents from LMICs reported that poor public awareness of the role of rehabilitation after stroke acted as a barrier to delivering evidencebased care in these settings. 
"Cultural barriers and religious beliefs regarding stroke and other forms of paralysis” [physiotherapist, India]

Some patients want to see rapid results, but rehabilitation is a long treatment and not accepted... many hospitals do not value rehabilitation [physician, China]

Links between clinical services and research or academic institutions were seen to facilitate guideline usage, and overall coordination of healthcare within a region could be either a facilitator if well-coordinated:

“Local and 'intense' networking between hospitals, primary care and municipal care in close collaboration with policy makers/county council/municipal council and patient organisations" [physician, Sweden]

or a barrier if care provision was fragmented.

"Health Department disbanded the Stroke Network so there is little ability to coordinate [evidence-based practice] efforts across the state. State Government funding and structural priorities do not always support best stroke care" [physiotherapist, Australia]

A final barrier reported was when rehabilitation was not funded appropriately. Healthcare system ... is fragmented with variable reimbursement processes that undervalue rehabilitation" [physician, USA]

Lack of financial support [physiotherapist, India]

\section{Comparison of factors influencing use of clinical practice guidelines in different countries}

We compared factors that were perceived to influence the use of guidelines and evidencebased practice between the five countries with the highest number of responses to the open- 
ended question, representing a mix of HICs (Australia, UK, Canada) and upper middleincome countries (Brazil, China) in five continents.

Consistent responses from these five regions were that a lack of time, a lack of funding, and issues relating to staffing (numbers, expertise) impacted negatively on health professionals' ability to deliver evidence-based care that adhered to guideline recommendations. Clinical champions and good leadership were reported as facilitators to guideline use in all regions other than China. Specific to individual countries, the national audit programs were seen as beneficial in Australia, the UK and Canada. Regional supports, such as stroke networks and communities of practice were also perceived positively by respondents from these three countries. A frequently reported barrier specific to China was patient and family expectations of rehabilitation were unrealistic, and acted as a barrier for implementing guideline recommendations. Barriers that were only reported by respondents from Brazil were overcrowded facilities, a poor health system and out-of-date guidelines.

\section{Discussion}

Locally endorsed guidelines were identified for 15 of the $21 \mathrm{HICs}$ and for 6 of the 8 LMICs represented in our sample. The majority of health professionals working in stroke rehabilitation from HICs were aware of their national stroke rehabilitation guidelines when these were available, although there were surprising anomalies with the US and Singapore. Health professionals from LMICs were generally less aware of their local guidelines.

The factors reported to influence use of guidelines varied, with factors regarding the inner setting most often reported. The range of factors influencing use of stroke rehabilitation guidelines across all domains reflects those found in previous work.[41]

Resource-related issues such as available time and staff were frequently reported as barriers to providing guideline-directed care. Given that a country's income level is 
associated with the ratio of rehabilitation health professionals to population, as well as the skills of health professionals, [12][42], it is unsurprising that resource-related issues were reported by respondents from LMICs. Yet, despite the comparative advantage in HICs, respondents from these regions also commonly reported that resource availability hindered their ability to deliver care consistent with stroke rehabilitation guidelines. It could be that even well-resourced health systems do not have adequate staffing levels to provide care that adheres with guideline recommendations. Alternately it could be that health professionals in HICs perceive implementation of guideline recommendations as over and above core business, thereby requiring extra resources.

Outside of resource issues, the importance of contextual factors, such as workplace culture and readiness to change, and their influence on effective delivery of interventions has been recognised by many implementation science researchers.[15, 43-45] Despite this, we know very little about how modifiable the local context is or how to change it. Strategies to support quality improvement are generally focussed on the specific interventions or service delivery initiatives, and are not designed to address the functioning of the local context itself.[46] In the first instance, being able to measure or define elements of the local context might be helpful and there have been efforts to develop contextual measurement tools.[47, 48] Our findings suggest that more work is required to develop and evaluate strategies that can influence or adapt to the local context, thereby addressing team processes and accountability to deliver guideline-directed care, which were deemed by our international respondents to influence stroke rehabilitation guideline use. Our findings suggest that simply increasing resources may not be effective in supporting implementation, if the local contextual barriers are not addressed.

Respondents commonly cited participation in education and training activities as facilitating guideline use. However, despite the common use of education and training, these 
strategies alone are not effective in changing the practice of health professionals working in stroke rehabilitation.[49] Rather, multicomponent interventions are needed, including facilitation and tailoring to the context.[49]

Research engagement and research active organisations have been shown to offer superior health service performance, a higher quality of care, improved patient safety and provide greater opportunities for staff development.[50, 51] Respondents to the current survey concurred that being involved in research or affiliated with research organisations facilitated their use of clinical practice guidelines.

The strengths of this work include participation from health professionals from 30 countries, albeit with uneven representation, which provides a broad international overview regarding stroke rehabilitation guideline use. This information provides a useful addition to previous work in this area, with concrete examples of factors to consider when aiming to increase guideline use and improve patient care. Use of the online survey tool was freely accessible, but limited the robustness of the data collected, with participants frequently responding to the open-ended questions in single sentences.

We used the CFIR as our determinant framework, which provided a structured way for considering the factors reported to influence guideline use. A recent publication suggested that if using in LMICs, it may be of use to add a "Characteristics of Systems" domain that characterizes broader health systems and geopolitical factors.[52] However, this was published after our analyses was complete, and we felt able to code all responses using the existing constructs and that each construct had salience. Previous authors have reported challenges with overlapping constructs.[53] We had similar issues e.g. relationships between teams and external organisations (clinical academic links could be regarding the inner or outer setting), resources (could be regarding the intervention or the inner setting). This was overcome by discussions amongst the authors and the use of concrete examples. 
In summary, the majority of international survey respondents had access to stroke rehabilitation guidelines, and a higher proportion of respondents from HICs reported awareness of their local guidelines. A lack of time, funding, and qualified staff were reported to reduce adherence to guideline recommendations from respondents from all world regions. Good leadership, clinical champions, and access to national audit data were reported to increase the use of guidelines and the delivery of evidence-based care. Processes reported to improve guideline use included education, training, formation of workgroups, audit-feedback cycles and workplace accountability. Strategies to develop, update and raise awareness of guidelines that address the local context are still needed to improve the use of guidelines in stroke rehabilitation internationally.

\section{Acknowledgements}

We would like to acknowledge Julie Bernhardt for convening the second Stroke Rehabilitation and Recovery Roundtable (SRRR II), Dale Corbett for organizing the meeting, and Farrell Leibovitch for moderating discussions. We also acknowledge the SRRR2 knowledge translation working group: Janice J Eng, Erin Godecke, Tammy C Hoffmann, Carole Laurin, Olumide A Olaoye, John Solomon, Robert Teasell, Caroline L Watkins, Marion F Walker.

\section{Author contributions}

MLB facilitated stakeholder input to the survey design, EAL and MLB conceived the analysis, EAL, LBC, LAC and MLB analysed and interpreted the data, all authors contributed to manuscript write-up, all authors have read and approved the manuscript.

\section{Declaration of interests}


The authors report no conflicting interests. EAL receives salary support from a National Health and Medical Research Council Early Career Fellowship (\#1138515).

The following financial support was received to conduct the SRRR II meeting: Canadian Institutes of Health Research (CIHR) CaSTOR (Canadian Stroke Trials for Optimized Results) Group (note that CasTOR is a joint initiative of the Canadian Stroke Consortium and the Canadian Partnership for Stroke Recovery), Heart and Stroke Canadian Partnership for Stroke Recovery, and NHMRC Centre of Research Excellence in Stroke Rehabilitation and Brain Recovery. An unrestricted educational grant was provided by Ipsen Pharma.

\section{Data availability statement}

The data that support the findings of this study are available from the corresponding author on reasonable request. 


\section{References}

1. GBD 2016 Stroke Collaborators. Global, regional and national burden of stroke, 1990-2016: a systematic analysis for the Global Burden of Disease Study 2016. Lancet Neurology. 2019;18:439-58. 2. Walker MF, Fisher RJ, Korner-Bitensky N, McCluskey A, Carey LM. From What We Know to What We Do: Translating Stroke Rehabilitation Research into Practice international Journal of Stroke. 2013;8:11-7.

3. Kings College London. Sentinel Stroke National Audit Program 2020 [Available from: https://www.strokeaudit.org/.

4. Stroke Foundation. National Stroke Audit - Rehabilitation Services Report 2020. Melbourne, Australia: Stroke Foundation; 2020.

5. Canadian Stroke Network. The quality of stroke care in Canada. 2011.

6. Glasziou P, Straus S, Brownlee S, Trevena L, Dans L, Guyatt G, et al. Evidence for underuse of effective medical services around the world. Lancet. 2017:[epub ahead of print].

7. Field M, Lohr K. Guidelines for clinical practice: from development to use. Washington: National Academy Press; 1992.

8. $\quad$ Shekelle PG, Woolf SH, Grimshaw J. Developing guidelines. BMJ. 1999;318:593.

9. Platz T. Evidence-based guidelines and clinical pathways in stroke rehabilitation - an international perspective. Frontiers in Neurology. 2019;10:200.

10. Gonzales-Suarez C, Grimmer-Somers KA, King E, Lorenzo S, Valdecanas C, Gambito E, et al. Contextualizing Western guidelines for stroke and low back pain to a developing country (Philippines): an innovative approach to putting evidence into practice efficiently. Journal of Healthcare Leadership. 2012;4:141-56.

11. Grimmer KA, Louw Q, Dizon JM, Brown S-M, Ernstzen D, Wiysonge CS. A South African experience in applying the Adopt-Contextualise-Adapt framework to stroke rehabilitation clinical practice guidelines. Health Research Policy and Systems. 2019;17:56.

12. Bernhardt J, Urimubenshi G, Gandhi DBC, Eng JJ. Stroke rehabilitation in low-income and middle-income countries: a call to action. Lancet. 2020;396:1452-62.

13. Bernhardt J, Hayward KS, Dancause N, Lannin NA, Ward NS, Nudo RJ, et al. A Stroke recovery trial development framework: Consensus-based core recommendations from the second Stroke Recovery and Rehabilitation Roundtable. Neurorehabilitation and Neural Repair. 2019;33:959-69.

14. Eng JJ, Bird M-L, Godecke E, Hoffmann TC, Laurin C, Olaoye OA, et al. Moving stroke rehabilitation research evidence into clinical practice: Consensus-based core recommendations from the Stroke Recovery and Rehabilitation Roundtable. International Journal of Stroke. 2019;14:766-73. 15. Damschroder LJ, Aron DC, Keith RE, Kirsh SR, Alexander JA, Lowery JC. Fostering implementation of health services research findings into practice: a consolidated framework for advancing implementation science. Implementation Science. 2009;4(1):50.

16. The World Bank Group. Countries and Economies 2020 [Available from: https://data.worldbank.org/country.

17. Stroke Foundation. Clinical Guidelines for Stroke Management. Melbourne, Australia: Stroke Foundation; 2017.

18. Ministry of Health. Guidelines for the rehabilitation of individual with cerebrovascular accident. Brazil: Ministry of Health, Health Mo; 2013.

19. Hebert D, Lindsay MP, McIntyre A, Kirton A, Rumney PG, Bagg S, et al. Canadian stroke best practice recommendations: Stroke rehabilitation practice guidelines, update 2015. International Journal of Stroke. 2016;11(4):459-84.

20. Chinese Medical Association. Guidelines for early stroke rehabilitation in China. Chinese Journal of Neurology. 2017;50(6).

21. Administrative Steering Group. Rehabilitation of adults with acquired brain damage. Denmark; 2018. 
22. Dohle C, Quintern J, Saal S, Stephan KM, Tholen R, Wittenberg H. S2e-Leitlinie Rehabilitation der Mobilität nach Schlaganfall (ReMoS). Neurologie und Rehabilitation. 2015;21:355-494.

23. Khurana D, Padma MV, Bhatia R, Kaul S, Pandian J, Sylaja PN, et al. Recommendations for the Early Management of Acute Ischemic Stroke: A Consensus Statement for Healthcare Professionals from the Indian Stroke Association. Journal of Stroke Medicine. 2018;1(2):79-113.

24. Irish Heart Foundation: Council for Stroke. National Clinical Guidelines and

Recommendations for the Care of People with Stroke and Transient Ischaemic Attack. Ireland: Irish Heart Foundation; 2010.

25. Stroke Prevention and Educational Awareness Diffusion (SPREAD). Stroke: Italian guidelines on prevention and treatment. Italy: SPREAD; 2016.

26. Latvian Society of Neurologists. Clinical guidelines for prehospital care, diagnosis and acute care of cerebral infarction. Latvia: Latvian Society of Neurologists; 2013.

27. Malaysian Society of Neurosciences. Clinical Practice Guidelines Management of Ischaemic stroke. Malaysia: Malaysian Society of Neurosciences; 2012.

28. Norwegian Directorate of Health. Stroke: National Academic Guidelines. Norway: Norwegian Directorate of Health; 2017.

29. Ministry of Public Health: State of Qatar. Clinical Guidelines for the State of Qatar: The diagnosis and management of stroke and transient ischemic attack. Doha, Qatar: Ministry of Public Health: State of Qatar; 2016.

30. Venketasubramanian N, Pwee KH, Chen CPL. Singapore Ministry of Health Clinical Practice Guidelines on Stroke and Transient Ischemic Attacks. International Journal of Stroke. 2011;6(3):2518.

31. Bryer A, Connor MD, Haug P, Cheyip B, Staub H, Tipping B, et al. The South African Guideline for the Management of Ischemic Stroke and Transient Ischemic Attack: Recommendations for a Resource-Constrained Health Care Setting. International Journal of Stroke. 2011;6(4):349-54.

32. Kim DY, Kim Y-H, Lee J, Chang WH, Kim M-W, Pyun S-B, et al. Korean Standard Practice Guideline for Stroke Rehabilitation Treatment 2016. Brain Neurorehabil. 2017;10(Suppl 1).

33. National Board of Health and Welfare. National guidelines for stroke care and management, updated 2020. Sweden: National Board of Health and Welfare; 2018.

34. Department of Nursing and Health Care: Ministry of Health and Welfare. Stroke assessment and care guidelines, updated 2020. China: Ministry of Health and Welfare; 2004.

35. National Netherlands General Practitioners Society. Stroke treatment standards. Netherlands: National Netherlands General Practitioners Society; 2017.

36. Rudd A, Bowen A, Young G, James $M$. The latest National clinical guideline for stroke. Clinical Medicine. 2017;17(2):154-5.

37. National Institute for Health and Care Excellence (NICE). Stroke rehabilitation in adults. NICE; 201312 June 2013.

38. Scottish Intercollegiate Guidelines Network (SIGN). Management of patients with stroke: Rehabilitation, prevention and management of complications, and discharge planning - A national clinical guideline. Scotland: SIGN; 2010.

39. Order of the Ministry of Health. Unified Clinical Protocol of Health Care - Ischemic Stroke (emergency, primary, secondary medical care, medical rehabilitation). Ukraine: Order of the Ministry of Health; 2012.

40. Winstein CJ, Stein J, Arena R, Bates B, Cherney L, Cramer SC, et al. Guidelines for Adult Stroke Rehabilitation and Recovery A Guideline for Healthcare Professionals From the American Heart Association/American Stroke Association. Stroke. 2016;47(6):E98-E169.

41. Baatiema L, Otim ME, Mnatzaganian G, de-Graft Aikins A, Coombes J, Somerset S. Health professionals' views on the barriers and enablers to evidence-based practice for acute stroke care: a systematic review. Implement Sci. 2017;12(1):74.

42. Chimatiro GL, Rhoda AJ. Scoping review of acute stroke care management and rehabilitation in low and middle-income countries. BMC Health Services Research. 2019;19:789. 
43. May C, Johnson M, Finch T. Implementation, context and complexity. Implementation Science. 2016;11:141.

44. Dopson S, Fitzgerald L. The active role of context. In: Dopson S, Fitzgerald L, editors. Knowledge to action? Evidence-based health care in context. New York: Oxford University Press; 2005. p. 79-103.

45. Harvey G, Kitson A. PARIHS revisited: from heuristic to integrated framework for the successful implementation of knowledge into practice. IMplementation Science. 2015;11:33.

46. Varkey P, Reller MK, Resar RK. Basics of Quality Improvement in Health Care. Mayo Clinic Proceedings. 2007;82(6):735-9.

47. Fernandez ME, Walker TJ, Weiner BJ, Calo WA, Liang S, Risendal B, et al. Developing measures to assess constructs from the Inner Setting domain of the Consolidated Framework for Implementation Research. Implementation Science. 2018;13(1):52.

48. Estabrooks C, Squires J, Cummings G, Birdsell J, Norton P. Development and assessment of the Alberta Context Tool. BMC Health Services Research. 2009;9:234.

49. Bird M-L, Miller T, Connell LA, Eng JJ. Moving stroke rehabilitation evidence into practice: a systematic review of randomized controlled trials. Clinical Rehabilitation. 2019;33(10):1586-95.

50. Jonker L, Fisher SJ. The correlation between National Health Service trusts' clinical trial activity and both mortality rates and care quality commission ratings: a retrospective cross-sectional study. Public Health. 2018;157:1-6.

51. Jonker L, Fisher SJ, Dagnan D. Patients admitted to more research - active hospitals have more confidence in staff and are better informed about their condition and medication: Results from a retrospective cross - sectional study. Journal of Evaluation in Clinical Practice. 2020;26(1):203-8. 52. Means AR, Kemp CG, Gwayi-Chore M-C, Gimbel S, Soi C, Sherr K, et al. Evaluating and optimizing the consolidated framework for implementation research (CFIR) for use in low- and middle-income countries: a systematic review. Implementation Science. 2020;15(1):17.

53. Damschroder L, Lowery J. Evaluation of a large-scale weight management program using the consolidated framework for implementation research (CFIR). Implementation Science. 2013;8(1):51. 
Table 1. Awareness and availability of guidelines from countries represented in sample

\begin{tabular}{|c|c|c|c|c|}
\hline Country & $\begin{array}{l}\text { Number of } \\
\text { respondents }\end{array}$ & $\begin{array}{l}\text { Income level } \\
\text { of country }\end{array}$ & $\begin{array}{l}\text { Aware of local stroke } \\
\text { rehabilitation guidelines n } \\
\text { (\% of country's respondents) }\end{array}$ & $\begin{array}{l}\text { Local stroke rehabilitation guidelines identified in online } \\
\text { search, available as at June } 2018 \text { (Language of publication if } \\
\text { other than English) }\end{array}$ \\
\hline Australia & 156 & high & $154(99)$ & 2017 Stroke Clinical Guidelines, Stroke Foundation*1 \\
\hline Belgium & 2 & high & $0(0)$ & No clinical practice guideline identified \\
\hline Brazil & 64 & middle & $46(72)$ & $\begin{array}{l}\text { Guidelines for the rehabilitation of individual with } \\
\text { cerebrovascular accident, Ministry of Health, } 2013 \\
(\text { Portuguese) }\end{array}$ \\
\hline Canada & 85 & high & $83(98)$ & $\begin{array}{l}\text { Canadian Stroke Best Practice Recommendations: Stroke } \\
\text { Rehabilitation Practice Guidelines, Update } 2015^{* 3}\end{array}$ \\
\hline China & 216 & middle & $184(85)$ & $\begin{array}{l}\text { Guidelines for early stroke rehabilitation in China, Chinese } \\
\text { Medical Association, } 2017 \text { (Chinese) }^{4}\end{array}$ \\
\hline Denmark & 1 & high & $1(100)$ & $\begin{array}{l}\text { Rehabilitation of adults with acquired brain damage, } \\
\text { Administrative Steering Group, } 2018 \text { (Danish) }\end{array}$ \\
\hline Germany & 7 & high & $7(100)$ & $\begin{array}{l}\text { Rehabilitation of mobility after Stroke (ReMoS) guideline, } \\
\text { ReMoS working group } 2015 \text { (German })^{6}\end{array}$ \\
\hline Greece & 1 & high & $0(0)$ & No clinical practice guideline identified \\
\hline India & 38 & middle & $15(39)$ & $\begin{array}{l}\text { Recommendations for the Early Management of Acute Ischemic } \\
\text { Stroke: A } \\
\text { Consensus Statement for Healthcare Professionals from the } \\
\text { Indian Stroke }\end{array}$ \\
\hline
\end{tabular}




\begin{tabular}{|c|c|c|c|c|}
\hline & & & & Association, $2018^{7}$ \\
\hline Ireland & 10 & high & $10(100)$ & $\begin{array}{l}\text { National Clinical Guidelines and Recommendations for the Care } \\
\text { of People with Stroke and Transient Ischaemic Attack, Irish } \\
\text { Heart Foundation: Council for Stroke, } 2010^{8}\end{array}$ \\
\hline Italy & 1 & high & $1(100)$ & $\begin{array}{l}\text { Stroke: Italian guidelines on prevention and treatment, Stroke } \\
\text { Prevention and Educational Awareness Diffusion, SPREAD, } \\
2016{\text { (Italian })^{9}}^{9}\end{array}$ \\
\hline Latvia & 1 & high & $1(100)$ & $\begin{array}{l}\text { Clinical guidelines for prehospital care, diagnosis and acute care } \\
\text { of cerebral infarction, Latvian Society of Neurologists, } 2013 \\
(\text { Latvian })^{10}\end{array}$ \\
\hline Malaysia & 3 & middle & $1(33)$ & $\begin{array}{l}\text { Clinical Practice Guidelines Management of Ischaemic stroke, } \\
\text { Malaysian Society of Neurosciences, } 2012^{11}\end{array}$ \\
\hline Nigeria & 7 & middle & $1(14)$ & No clinical practice guideline identified \\
\hline Norway & 3 & high & $3(100)$ & $\begin{array}{l}\text { Stroke: National Academic guidelines, Norwegian Directorate of } \\
\text { Health, } 2017^{12}\end{array}$ \\
\hline Oman & 1 & high & $0(0)$ & No clinical practice guideline identified \\
\hline Portugal & 1 & high & $0(0)$ & No clinical practice guideline identified \\
\hline Qatar & 1 & high & $1(100)$ & $\begin{array}{l}\text { Clinical Guidelines for the State of Qatar. The diagnosis and } \\
\text { management of stroke and transient ischemic attack, Ministry of } \\
\text { Public Health, State of Qatar, } 2016^{13}\end{array}$ \\
\hline Saudi Arabia & 1 & high & $0(0)$ & No clinical practice guideline identified \\
\hline Singapore & 30 & high & $10(33)$ & $\begin{array}{l}\text { Clinical practice guidelines - Stroke and Transient Ischaemic } \\
\text { Attacks, Ministry of Health Singapore, } 2011^{14}\end{array}$ \\
\hline
\end{tabular}




\begin{tabular}{|c|c|c|c|c|}
\hline South Africa & 2 & middle & $1(50)$ & $\begin{array}{l}\text { South African guideline for management of ischaemic stroke } \\
\text { and transient ischaemic attack: A guideline from the South } \\
\text { African Stroke Society (SASS) and the SASS Writing } \\
\text { Committee, } 2010^{15}\end{array}$ \\
\hline South Korea & 3 & high & $3(100)$ & $\begin{array}{l}\text { Clinical Practice Guideline for Stroke Rehabilitation in Korea, } \\
2016(\text { Korean })^{16}\end{array}$ \\
\hline Sudan & 1 & low & $0(0)$ & No clinical practice guideline identified \\
\hline Sweden & 14 & high & $14(100)$ & $\begin{array}{l}\text { National guidelines for stroke Care and management, National } \\
\text { Board of Health and Welfare, 2017-2018 (Swedish)*17 }\end{array}$ \\
\hline Taiwan & 4 & high & $3(75)$ & $\begin{array}{l}\text { Stroke assessment and care guidelines, Department of Nursing } \\
\text { and Health Care, Ministry of Health and Welfare, last version } \\
2004 \text { (Chinese) } \text { *1 }^{18}\end{array}$ \\
\hline $\begin{array}{l}\text { The } \\
\text { Netherlands }\end{array}$ & 4 & high & $3(75)$ & $\begin{array}{l}\text { Stroke treatment standards, National Netherlands General } \\
\text { Practitioners Society, } 2017 \text { (Dutch) })^{19}\end{array}$ \\
\hline $\begin{array}{l}\text { United Arab } \\
\text { Emirates }\end{array}$ & 1 & high & $0(0)$ & No clinical practice guideline identified \\
\hline $\begin{array}{l}\text { United } \\
\text { Kingdom (other } \\
\text { than Scotland) } \\
\text { Scotland }\end{array}$ & 90 & high & $35(100)$ & $\begin{array}{l}\text { National Clinical guideline for stroke, Royal College of } \\
\text { Physicians, } 2016^{20} \\
\text { Stroke rehabilitation in adults, NICE clinical guideline, } 2013 ;^{21} \\
\text { Management of patients with stroke: Rehabilitation, prevention } \\
\text { and management of complications, and discharge planning - A }\end{array}$ \\
\hline
\end{tabular}




\begin{tabular}{|l|l|l|l|l|}
\hline & & & & $\begin{array}{l}\text { national clinical guideline, Scottish Intercollegiate Guidelines } \\
\text { Network (SIGN), 2010 }\end{array}$ \\
\hline Ukraine & 4 & middle & $2(50)$ & $\begin{array}{l}\text { Unified Clinical Protocol of Health Care - Ischemic Stroke } \\
\text { (emergency, primary, secondary medical care, medical } \\
\text { rehabilitation) - Order of the Ministry of Health, 2012 } \\
(\text { Ukrainian })^{23}\end{array}$ \\
\hline $\begin{array}{l}\text { United States of } \\
\text { America }\end{array}$ & 46 & high & $43(93)$ & $\begin{array}{l}\text { AHA/ASA Guidelines for Adult Stroke Rehabilitation and } \\
\text { Recovery, 2016 }\end{array}$ \\
\hline
\end{tabular}

